\section{Intersections}

Canadian Journal of Music

Revue canadienne de musique
Intersections CANADIAN JOURAL OR MUSIO

\title{
La réception des productions culturelles rock : étude comparée entre la presse musicale québécoise et française
}

\section{Fabien Hein}

Volume 25, numéro 1-2, 2005

URI : https://id.erudit.org/iderudit/1013303ar

DOI : https://doi.org/10.7202/1013303ar

Aller au sommaire du numéro

Éditeur(s)

Canadian University Music Society / Société de musique des universités canadiennes

ISSN

1911-0146 (imprimé)

1918-512X (numérique)

Découvrir la revue

Citer cet article

Hein, F. (2005). La réception des productions culturelles rock : étude comparée entre la presse musicale québécoise et française. Intersections, 25(1-2), 23-39. https://doi.org/10.7202/1013303ar
Résumé de l'article

À partir d'une étude comparative chiffrée, cet article vise à porter un regard croisé sur la réception accordée par la presse musicale française aux productions rock québécoises/canadiennes, et inversement, à la réception accordée par la presse musicale québécoise aux productions rock françaises. À condition de ne pas séparer les données obtenues des réalités du travail de la réception critique tout comme de la circulation des productions culturelles, cette démarche permet d'évaluer la dynamique des échanges culturels entre les deux aires francophones.
Copyright (C Canadian University Music Society / Société de musique des universités canadiennes, 2005
Ce document est protégé par la loi sur le droit d'auteur. L'utilisation des services d'Érudit (y compris la reproduction) est assujettie à sa politique d'utilisation que vous pouvez consulter en ligne.

https://apropos.erudit.org/fr/usagers/politique-dutilisation/ 


\section{LA RÉCEPTION DES PRODUCTIONS CULTURELLES ROCK : ÉTUdE COMPARÉE ENTRE LA PRESSE MUSICALE QUÉBÉCOISE ET FRANÇAISE ${ }^{1}$}

\section{Fabien Hein}

\section{INTRODUCTION}

Plusieurs études ont permis de démontrer avec profit combien la presse musicale, en tant que dispositif critique local, constituait un instrument de mesure efficace pour analyser les discours ayant trait à certaines productions culturelles ou à certains artistes " rock $^{2}$ " (André 1993; Willey 1994; Rudent 2000; Teillet 2002). Toutefois, nous ne savons que très peu de choses quant aux modalités concrètes de réception que cette presse accorde aux productions rock (disques, artistes, fanzines, concerts, etc.). Pour explorer cette question, je propose d'employer une méthode comparative conjointement articulée à une pratique de dénombrement systématique. J'observerai deux dispositifs critiques issus d'espaces géoculturels distincts : la presse rock française et la presse rock québécoise d'expression francophone. De cette manière, j'entends porter un regard croisé sur la réception accordée par la presse musicale française aux productions rock québécoises/canadiennes, et inversement, à la réception accordée par la presse musicale québécoise aux productions rock françaises. Une démarche qui permettra d'évaluer la dynamique des échanges culturels entre les deux aires francophones.

Â ma connaissance, il n'existe pas pour l'heure de recherches analogues. Toutefois, les échanges culturels entre la France et le Québec ont été étudiés sous d'autres dimensions. On dispose par exemple d'études abordant des questions aussi variées que les échanges intellectuels (Fabre 2004; Angers et Fabre 2004), les échanges littéraires (Vincent 1997; Casanova 1999), les échanges thêatraux (Paoli 1993) ou encore les échanges musicaux (De Surmont 1996). Chacune de ces études souligne la fécondité incontestable de la démarche comparatiste qu'Emile Durkheim a érigé en règle de méthode sociologique dès 1894 . Succès d'ailleurs régulièrement confirmé par un cer-

1 Je tiens à remercier la Bibliothèque Nationale du Québec à Montréal pour m'avoir permis de réaliser cette étude comparée. Mes remerciements vont également à Serge Lacasse et à Sandria $P$. Bouliane.

2 Le « rock " est une catégorie des musiques populaires. On situe communément ses origines au milieu des années 1950 sous l'impulsion d'Elvis Presley. Il s'agit d'un terme générique, hétérogène et dynamique permettant de désigner, peu ou prou, une multitude de genres musicaux apparentés (du rock'n'roll au heavy metal en passant par le punk, etc.), mais également un ensemble complexe de pratiques artistiques et culturelles articulant acteurs, objets et dispositifs affiliés. 
tain nombre de travaux théoriques visant à valoriser les études comparées (Sartori 1994; Langlois 2002; Vigour 2005). Dans ce prolongement, ma démonstration articule trois conditions interdépendantes :

1. Contextualisation : le monde du rock transnational.

2. Assimilation : la presse musicale rock au Québec et en France.

3. Différenciation : la réception des productions rock au sein de cette presse musicale.

Ce préalable méthodologique permet de structurer mon étude comparative. Ainsi, le monde du rock transnational fixe le contexte général de ma recherche. Il englobe la presse musicale rock québécoise et française qui, par assimilation, autorise le travail de comparaison. Opération nécessitant dans le même temps un travail de différenciation par rapport au critère de la réception envisagé au sein de cette presse musicale.

Le rock est un produit artistique et culturel universel, sinon transfrontalier. Il s'épanouit dans les pays industrialisés ou, à tout le moins, desservis en électricité. D'un point de vue historique, il est né aux États-Unis qui en a été l'épicentre pendant les années 1950, avant de se déplacer en Grande-Bretagne au cours des années 1960. À la suite de quoi, la prolifération des genres aidant, les pôles de production majeurs du rock se sont surtout déplacés en fonction de "scènes musicales" (Straw 1991) spécifiques, chacune d'entre elles étant constitutive du monde du rock. Ces scènes sont généralement localisées au sein de villes (la scène grunge de Seattle, la scène post rock de Montréal, la scène death metal mélodique de Göteborg, etc.), ou au sein de pays (la scène krautrock en Allemagne, la scène punk en Grande-Bretagne, la scène black metal en Norvège, etc.). Par conséquent, il est impossible d'identifier un méridien de Greenwich propre au monde du rock à la façon dont Pascale Casanova (1999) a pu le faire pour le monde de la littérature. Cette fragmentation indique que le monde du rock est en fait un monde pluriel et fortement différencié. Ce qui constitue une caractéristique forte pour mon étude. Après avoir défini ce que j'entends par " monde du rock " et par " presse musicale ", je procéderai à la description de mon échantillon avant d'entamer le travail de comparaison qui constitue l'objet de cet article.

\section{LE MONDE DU ROCK}

La spécificité d'un monde est d'être produit et de produire symétriquement les acteurs et les objets en tant qu'ils sont à la fois constitutifs et participants de ce monde. Autrement dit, il résulte d'un processus de médiations entre des humains et des choses assurant le fonctionnement de ce monde (Hennion 1993; Hein 2006). Au Québec comme en France, le monde du rock procède d'un certain nombre d'activités de production réunissant acteurs, objets et dispositifs. L'observation spécifiquement centrée sur des objets tels que les périodiques et les disques permet de retracer le chemin réticulaire qui mène aux acteurs et aux dispositifs ${ }^{3}$. Les périodiques sont produits par un appareil

3 Ma posture sociologique s'inscrit clairement dans le sillage d'une sociologie des sciences, telle 
de commentaires constitué de prescripteurs. Les disques sont quant à eux produits par des musiciens et des producteurs discographiques. Généralement, tous ces acteurs ont en commun d'être des amateurs de rock s'adressant à d'autres amateurs de rock. Ensemble, ils constituent des médiateurs culturels dont la contribution permet l'existence du monde du rock. Comme j'ai déjà pu le mentionner plus haut, le monde du rock se partage en une multitude de "scènes musicales » constituées sur la base d'une communauté de goût. Ces scènes sont généralement de trois types, comme les travaux d'Andy Bennett et de Richard Peterson (2004) ont pu le souligner. On trouve des scènes locales, des scènes translocales et des scènes virtuelles parfaitement poreuses les unes aux autres.

\section{La presse musicale au Québec et en France}

La presse papier spécialisée rock se partage généralement entre une presse " magazine " à diffusion nationale et une presse " fanzine " nettement plus confidentielle. Les magazines sont des publications élaborées pour des amateurs par des acteurs qui en font profession. Elles ont des buts commerciaux, sont produites par une équipe de journalistes rémunérés, font de la publicité, utilisent des moyens de diffusion conséquents ${ }^{4}$ et touchent un public important. Les fanzines sont quant à eux des publications produites sur le temps de loisir par et pour les amateurs. Leurs rédacteurs revendiquent des valeurs de plaisir, de partage, de créativité, etc., mais ne visent pas explicitement la réalisation d'un profit. Selon Samuel Étienne $(2003,11)$, le fanzine " représente un mode de communication horizontal nivelé et bidirectionnel (les fans parlent directement aux autres fans et ceux-ci ont la possibilité de répondre), alors que la presse dominante est un mode de communication vertical hiérarchisé et descendant (les professionnels - sous entendus "spécialistes" autoproclamés - parlent aux profanes qui n'ont pas ou peu de possibilité de répondre) ". Les deux types de publications rendent cependant les mêmes services : l'évaluation de la qualité d'une production et la conservation de cette production (Hein 2003b).

En France, depuis ses origines dans les années 1960, la presse musicale de format magazine semble être relativement pérenne en dépit d'une instabilité chronique des titres ${ }^{5}$. Les fanzines, par nature éphémères, sont eux aussi très florissants ${ }^{6}$. Les deux types de publication ont en commun de se positionner à la fois sur des terrains musicaux généralistes et sur des terrains musicaux ultra spécialisés, avec toutefois une prédilection pour la seconde catégorie.

qu'elle a été stabilisée par Bruno Latour (1989), Michel Callon (1986) et, pour ce qui concerne plus spécifiquement la musique, Antoine Hennion (1993).

4 En France, par exemple, la diffusion est essentiellement assurée par les NMPP (Nouvelles messageries de la presse parisienne), qui distribuent près de $90 \%$ de la presse vendue sur le territoire.

5 On dénombre actuellement environ une dizaine de titres en activité parmi lesquels : Rock 6 Folk, Rock Sound, Elegy, D-Side, Rock Hard, Metallian, Hard N'Heavy, Versus, Velvet, etc. La majorité d'entre eux sont disponibles dans les librairies québécoises.

6 En juin 2000, la Fanzinothèque de Poitiers recense 1500 titres de fanzines français et francophones. Voir http://www.fanzino.com/docrok/docsommaire.htm (consulté le 15 juillet 2005). 
Longueur d'Ondes, "magazine dédié aux musiques nouvelles et à la culture rock de l'espace francophone " tel qu'il se définit lui-même, apparaît comme le seul périodique français accordant une attention spécifique, soutenue et systématique aux productions québécoises. L'identité de la publication est ici très clairement marquée par un intérêt pour les productions françaises et québécoises. Elle est inverse aux autres publications qui ne se définissent jamais aussi explicitement en référence à des pôles de production aussi localisés.

Au Québec, la presse rock de format magazine, essentiellement généraliste, s'est épanouie du début des années 1970 à la fin des années 1980 avant de se retirer définitivement du paysage de la presse musicale ${ }^{7}$, cédant ainsi progressivement la place aux fanzines, plus spécialisés, dont les premiers titres font leur apparition dans la première moitié des années $1980^{8}$. Si bien que la presse musicale francophone au Québec n'est plus représentée aujourd'hui que par une poignée de fanzines ${ }^{9}$, signe que la presse commerciale n'y est manifestement pas très rentable d'un point de vue économique ${ }^{10}$.

\section{DESCRIPTION DE L'ÉCHANTILLON}

Très concrètement, cette étude repose sur un échantillon de quatre périodiques : deux périodiques français - Rock ఓFolk et Rock Hard - et deux périodiques québécois - Live! et Sang Frais. La constitution de ce corpus mérite quelques précisions. Il a évidemment été conçu, autant que faire se peut, sur la base d'un certain nombre de caractéristiques communes et opposées permettant de les comparer. Dans une première configuration à dimension globale, ces périodiques ont en commun d'appartenir au monde du rock et de s'opposer quant à leur aire de diffusion géographique. Dans une seconde configuration, Rock $b$ Folk et Live! se rejoignent à la fois sur une ligne éditoriale généraliste et sur leur longévité qui leur confère un statut singulier dans l'histoire de la presse rock francophone ${ }^{11}$. Ils s'opposent à Rock Hard et

7 Par ordre de parution, on comptait dans les années 1970 : Pop Jeunesse, Pop Jeunesse Rock, Pop Rock, Québec Rock, Vedette Rock, Vibrations; et dans les années 1980 : Live!, Vedettes Rock.

8 Par ordre de parution, on comptait dans les années 1980 : Le Monde du Rock, Metal K.O., Mega Thrash, Lezzzard; et dans les années $1990:$ L'Inconnu, Cyclone, Pandémonium, Kérozen et L'Édition Metallique. À ce jour, tous ces titres ont cessé de paraître.

9 Les plus importantes de ces publications se présentent elles-mêmes toujours en tant que magazines. Elles ont un tirage supérieur ou équivalent à 5000 exemplaires. Toutefois, sachant qu'elles ne bénéficient pas d'un circuit de distribution commercial et qu'elles ne disposent que rarement d'un personnel salarié, il est impossible de les considérer en tant que tels. Il me semble plus pertinent de les considérer en tant qu'objet hybride tenant à la fois du fanzine et du magazine. Par commodité langagière, j'utiliserai le terme fanzine à leur endroit. Par ordre de parution, on trouve Emoragei, Rien à Déclarer, Sang Frais, Bazooka.

$10 \AA$ Aitre d'illustration, on peut lire la chose suivante dans Rien à Déclarer, $\mathrm{n}^{\circ} 20$, printemps 2005 : "On recherche journaliste : efficacité à faire des entrevues sur commande, ponctualité, bilingue, bien connaître la scène locale et ou internationale tout style confondu est un atout. Recherche surtout dans le coin de Québec. Travail bénévole ". C'est moi qui souligne.

11 Bien que Live! ne soit plus en activité à l'heure actuelle, contrairement à RockઐFolk, il n'en est pas moins son seul équivalent québécois si on considère déterminant le critère de la longévité. 
Sang Frais qui ont pour traits communs de s'inscrire dans une ligne éditoriale spécialisée dans la scène metal ${ }^{12}$, mais aussi par leur indépendance à l'égard d'un groupe de presse. On voit que ces configurations peuvent se décliner sous des formes multiples pour les besoins de la démonstration ${ }^{13}$. Pour ce qui me concerne, avant de procéder à des comparaisons internationales, je comparerai tout d'abord les périodiques d'une même aire culturelle entre eux. À la suite de quoi, je chercherai à restituer les données obtenues dans leur environnement : celui du travail de la réception critique et celui de la circulation des productions culturelles.

\section{Périodiques musicaux français}

\section{RockeFolk}

Le mensuel RockઐFolk, né en 1966, est une institution de la presse musicale hexagonale de type généraliste. Il est le premier et le plus ancien magazine rock français en activité. Sa version "Nouvelle génération " est aujourd'hui dirigée par l'inénarrable Philippe Manœuvre (1985). De façon opératoire, j'ai étudié un échantillon de 36 numéros de RockßFolk correspondant à trois années de parution (janvier 2000 à décembre 2002). Il s'en dégage plusieurs éléments significatifs, dont les suivants :

- La majorité des informations concernant les productions canadiennes se trouve dans la rubrique "Chroniques albums". Les informations concernant les productions québécoises sont deux fois moins nombreuses que celles concernant les productions non québécoises.

- Trois artistes canadiens ont été interviewés. Parmi eux, Alanis Morissette et Hawksley Workman (Ontario), de même que Godspeed You! Black Emperor (Québec anglophone).

12 Pour avoir un panorama historique de la très fragmentée et imposante scène metal, voir Hein (2003a, chap. 2).

13 On pourra éventuellement s'étonner de la relative hétérogénéité du volume de périodiques consultés. De la même manière qu'on pourra également s'étonner de la relative disparité temporelle du corpus. Il est à ce titre important de noter que la recherche de l'homogénéité pour la constitution d'un corpus est parfois un objectif inatteignable et de fait, conduit à quelques compromis. Par exemple, mon ambition initiale visait à travailler sur des matériaux récents couvrant les années 2000 . Or, il n'existe pas de magazine généraliste québécois couvrant cette période. Il me faut donc accepter que mon échantillon compte trois périodiques couvrant les années 2000 pour un périodique couvrant les années 1980. Cet intervalle est-il préjudiciable à la qualité de mon étude? Je ne le pense pas, dans la mesure où la période constitue à mon sens un critère nettement moins déterminant que la ligne éditoriale ou la dimension historique du périodique dans le cas de RockéFolk et Live! par exemple. Par son unicité et sa longévité, Sang Frais bénéficie lui aussi d'un statut historique au sein de la presse musicale québécoise; statut auquel Rock Hard ne peut pas prétendre en France. À l'échelle du corpus, on observe donc là aussi un déséquilibre avec, d'un côté, trois figures historiques de la presse musicale et, de l'autre, un périodique moins prestigieux. Est-ce pour autant un critère rédhibitoire? Non, car parmi les multiples titres de la presse metal en France, Rock Hard est probablement celui qui s'apparente le plụs à Sang Frais, notamment pour son caractère indépendant. Ces éléments d'explication devraient permettre de neutraliser les distorsions propres à l'échantillon retenu, lequel, j'en conviens, est imparfait, mais aussi le plus ajusté possible à la réalité concrète du marché de la presse musicale. 
- Treize chroniques de disques canadiens ont été réalisées. Elles concernent les albums de neufs artistes non québécois (Neil Young, The Black Halos, Sum 41, Avril Lavigne, Alanis Morissette, Ron Sexsmith, etc.) et de deux artistes québécois anglophones (A Silver Mt Zion et Leonard Cohen pour plusieurs albums).

- Deux formations françaises, Bérurier Noir et Louise Attaque, ont été interviewées dans le cadre de leur tournée au Québec.

- Sont mentionnés dans les pages du mensuel à des titres divers, quatre artistes québécois originaires de Montréal : Godspeed You! Black Emperor, A Silver Mt Zion, Leonard Cohen (côté anglophone) et Grimskunk (côté francophone).

- Sont mentionnés dans les pages du mensuel et à des titres divers, neuf artistes canadiens non québécois, parmi lesquels Kittie, Neil Young, The Black Halos, Sum 41, Alanis Morissette, Ron Sexsmith, Hawksley Workman, Avril Lavigne et Destroyer.

- Les artistes canadiens les plus présents dans les pages du mensuel sont, par ordre d'importance, Léonard Cohen, Alanis Morissette et Hawksley Workman.

- Au total, sur 36 numéros de RockeFolk étudiés (4 680 pages), les artistes canadiens bénéficient d'un taux de présence ${ }^{14}$ de $0,57 \%$. Les artistes québécois occupent quant à eux un taux de présence de $0,14 \%$ et leur langue d'expression artistique est très majoritairement l'anglais. Il n'est pas nécessaire de s'appesantir sur ces résultats tant ils sont explicites ${ }^{15}$. De manière à en vérifier la pertinence, j'ai procédé à un travail analogue à partir d'un magazine non généraliste, spécialisé dans le metal.

\section{Rock Hard}

Le mensuel Rock Hard est l'un des titres de la bouillonnante presse metal française. Né courant 2001, son équipe de rédaction se compose de plusieurs pionniers de la presse metal française apparue au début des années $1980^{16}$. Contrairement aux autres périodiques du même type, Rock Hard se caractérise par sa totale indépendance à l'égard d'un groupe de presse quelconque. De façon opératoire, j'ai pris appui sur l'ensemble de la collection parue entre juin 2001 et avril 2005, ce qui représente 42 numéros. Il en ressort les éléments significatifs suivants :

14 Par " taux de présence ", il faut entendre un rapport entre le nombre de pages consultées et le nombre d'occurrences pertinentes. Chaque mention d'un artiste ou d'un disque canadien, québécois ou français constitue une occurrence.

$15 \AA$ la décharge de Rockefolk, il faut noter que le groupe montréalais Arcade Fire figure en première de couverture de l'édition du mois de septembre 2005 ( $\mathrm{n}^{\circ}$ 457). Rappelons également que la scène rock montréalaise connaît actuellement un engouement médiatique spectaculaire. En effet, des institutions telles que Rolling Stone, Spin Magazine et le New York Times ont décrété récemment que Montréal était la nouvelle capitale du rock. On ne peut qu'être amusé par ce type de déclaration à l'emporte-pièce qui souligne combien le discours médiatique dominant est à la fois normatif et réducteur, tout en soulignant avec force, et de manière involontaire, son aveuglement.

16 Pour un historique de la presse metal en France, se reporter à Hein (2003a, 204). 
- La majorité des informations concernant les productions canadiennes se trouve dans les rubriques "Chroniques albums" et "News ". Les informations concernant les productions québécoises sont deux à trois fois moins nombreuses que celles concernant les productions non québécoises.

- Sur 600 titres du sampler ${ }^{17}$ Rock Hard, quinze d'entre eux proviennent de formations canadiennes (Annihilator, Devin Townsend, Danko Jones, Harem Scarem, Strapping Young Lad et Exciter), dont deux sont d'origine québécoise (Liva et Voivod).

- Sur 16 artistes canadiens interviewés, deux d'entre eux sont d'origine québécoise : Slaves On Dope et Kataklysm.

- Les journalistes de Rock Hard ont assisté à 16 concerts de groupes canadiens en France. Parmi eux, on dénombre deux groupes québécois : Grimskunk et Kataklysm.

- 50 quatre chroniques de disques canadiens ont été rédigées. Elles concernent sept formations québécoises (Voivod en tête) et 25 formations non québécoises.

- Les trois artistes canadiens les plus présents dans les pages de Rock Hard sont par ordre d'importance : Devin Townsend (26), Annihilator (22) et Voivod (15). Ils sont suivis de Sum 41 (13), Rush (12), Strapping Young Lad $\left(10^{18}\right)$ et Danko Jones (10).

- Parmi les 25 artistes canadiens cités au moins à deux reprises dans l'ensemble de la collection étudiée, six d'entre eux sont d'origine québécoise francophone (Voivod, Kataklysm, Liva, Gorguts, Cryptopsy et Céline Dion ${ }^{19}$ ) et l'un d'entre eux est d'origine québécoise anglophone (Slaves On Dope). Leur langue d'expression artistique est majoritairement l'anglais.

- Au total, sur 42 numéros de Rock Hard étudiés (4 872 pages), les artistes canadiens bénéficient d'un taux de présence de $4,16 \%$. Les artistes québécois occupent quant à eux un taux de présence de $0,70 \%$ et leur langue d'expression artistique est très majoritairement l'anglais.

\section{COMPARAISON DES PÉRIODIQUES FRANÇAIS}

La comparaison des deux périodiques français permet de dégager plusieurs points importants. Il apparaît de manière évidente que Rock Hard accorde davantage d'intérêt aux productions canadiennes que son confrère Rock -Folk. Par ailleurs, les deux périodiques nourrissent un intérêt commun pour les trois artistes canadiens que sont Sum 41, Kittie et Grimskunk. D'autre part, la prédominance des artistes non québécois est observable au sein

17 Un sampler est un $C D$ promotionnel accompagnant le magazine.

18 Si l'on tient compte du fait que Devin Townsend est également le leader de Strapping Young Lad, on retrouve ce dernier à 36 reprises dans le magazine. Le fait qu'il soit un proche du rédacteur en chef de Rock Hard n'y est probablement pas étranger.

19 Pour surprenante qu'elle soit, la présence de Céline Dion s'explique par sa reprise du titre "You Shook Me All Night Long " d'AC/DC. 
des deux périodiques. De façon plus générale, les données obtenues indiquent la faiblesse de la réception des productions canadiennes et plus spécifiquement québécoises au sein des deux périodiques hexagonaux. Les résultats sont éloquents. Il s'agit maintenant d'inverser la problématique pour examiner si cette tendance se vérifie également en terme de réception des productions françaises au Québec.

\section{Périodiques MUSICAUX QUÉbécoIS}

\section{Live!}

Le trimensuel Live! "Le journal rock » est comparable à RockઐFolk en raison de son caractère généraliste. Bien qu'il ait cessé de paraître, le périodique détient un record de longévité au sein de la presse rock québécoise. Sa carrière couvre près d'une décennie. Il est en outre le seul dispositif critique québécois en activité au cours des années 1980. De façon opératoire, j'ai étudié l'ensemble de la collection parue entre mai 1980 et mars 1989; soit 137 numéros desquels se dégagent plusieurs éléments significatifs :

- La majorité des informations concernant les productions françaises se trouve dans la rubrique "News".

- Six artistes français ont été interviewés ou bénéficient d'un article : Catherine Lara, Indochine, Trust, Bijou, Téléphone et Edith Nylon.

- Les journalistes de Live! ont assisté à deux concerts de groupes français : Indochine et Rita Mitsouko.

- Quatre albums français ont été chroniqués, parmi lesquels deux albums de Jean-Michel Jarre et ceux de Jean-Jacques Goldman et Sortilège.

- Sur deux référendums des lecteurs, aucun groupe français n'est mentionné et on y trouve seulement trois artistes québécois. Sur cinq référendums des journalistes, seul Jean-Michel Jarre est cité une fois et on y trouve seulement deux artistes québécois.

- Parmi les 22 artistes français mentionnés, on retrouve le plus fréquemment, et par ordre d'importance, Sortilège (7), H-Bomb (6), Warning et Jean-Michel Jarre (5), Trust, Vulcain et Blasphème (4), Demon Eyes et Satan Jokers (3), Bijou, Rita Mitsouko et Indochine (2).

- Au total, sur 137 numéros de Live! étudiés (3 699 pages), les artistes français bénéficient d'un taux de présence de $1,54 \%$. Leur langue d'expression artistique est très majoritairement le français.

\section{Sang Frais}

Le trimestriel Sang Frais est comparable à Rock Hard en raison d'une ligne éditoriale exclusivement dédiée au metal. En activité depuis 1998, le périodique représente la formule la plus aboutie dans le genre au Québec, autant en terme de qualité que de régularité. Son autre spécificité tient au fait qu'il est, comme il se présente lui-même, "Le seul magazine metal francophone en Amérique ». De façon opératoire, j'ai étudié l'ensemble de la collection paru entre juin 1998 et juin 2005; soit 17 numéros desquels se dégagent les éléments significatifs suivants : 
- La majorité des informations concernant les productions françaises se trouve dans la rubrique "Chroniques albums», suivi de la rubrique «Fanzines".

- 57 albums d'artistes français ont été chroniqués, de même que 30 fanzines.

- Deux artistes français ont été interviewés : Anorexia Nervosa et Inhumate.

- Les journalistes de Sang Frais ont assisté à sept concerts de groupes français dont six au Québec et l'un en Belgique.

- Au printemps 2001, Sang Frais intègre une rubrique "CD français" dans les colonnes du numéro 8 , suivie dans le numéro 9 (été 2001) d'une rubrique intitulée "Du côté de la France " pour y présenter quelques nouvelles. Aujourd'hui, les chroniques de disques français sont surmontées du symbole FR, au même titre que les disques québécois sont accompagnés d'une fleur de lys et les disques canadiens d'une feuille d'érable.

- Parmi les 57 artistes français mentionnés dans les pages du trimestriel, on retrouve le plus fréquemment, et par ordre d'importance, Inhumate (5), Anorexia Nervosa et Misanthrope (3), Mass Hysteria, Lofofora et Rest In Peace (2).

- Parmi les 22 fanzines français mentionnés, on retrouve le plus fréquemment, et par ordre d'importance, O3 et La Lettre de Sappho (3), Decibels Storm, No Noise No Good, Underground Investigation et Zu Shan (2).

- Au total, sur 17 numéros de Sang Frais étudiés (850 pages), les productions françaises bénéficient d'un taux de présence de $10,70 \%$. Les fanzines sont tous d'expression française. Concernant les formations musicales, leur langue d'expression artistique est très majoritairement l'anglais.

\section{Comparaison DES PÉRIOdIQUES QUÉbÉCOIS}

La comparaison des deux périodiques québécois permet de dégager plusieurs points importants. L'intérêt que manifeste Sang Frais pour les productions françaises est sans commune mesure avec celui de Live! La quantité de disques français chroniqués en constitue une première preuve tangible. La symbolisation du logo FR en constitue une seconde. L'intérêt pour les fanzines en constitue une troisième. Toutefois, toute proportion gardée, il est intéressant de constater que Live! privilégie les formations d'expression française, contrairement à Sang Frais qui se montre davantage intéressé par des formations d'expression anglaise. Par ailleurs, les deux périodiques n'appartenant pas à la même génération, il n'est guère étonnant qu'elles ne nourrissent aucun intérêt commun pour des artistes particuliers.

\section{COMPARAISON DES PÉRIOdIQUES FRANÇAIS ET QUÉbÉCOIS}

Elargissons maintenant la comparaison à un niveau international à l'aide du tableau 1 ci-dessous. Je rappelle que le " taux de présence " représente un 
rapport entre le nombre de pages consultées et le nombre de mentions pertinentes d'un artiste ou d'un disque canadien, québécois ou français.

Tableau 1. Comparatif des taux de présence des productions québécoises et canadiennes dans la presse musicale française avec les productions françaises dans la presse musicale québécoise

\begin{tabular}{||c|c|c|c|c|c|c|}
\hline \multicolumn{1}{|c|}{ Titre } & $\begin{array}{c}\text { Nombre de } \\
\text { numéros } \\
\text { consultés }\end{array}$ & Période & $\begin{array}{c}\text { Somme des } \\
\text { pages } \\
\text { consultées }\end{array}$ & $\begin{array}{c}\text { Pourcentage } \\
\text { de présence: } \\
\text { productions } \\
\text { canadiennes }\end{array}$ & $\begin{array}{c}\text { Pourcentage } \\
\text { de présence : } \\
\text { productions } \\
\text { québécoises }\end{array}$ & $\begin{array}{c}\text { Pourcentage } \\
\text { de présence: } \\
\text { productions } \\
\text { françaises }\end{array}$ \\
\hline \hline $\begin{array}{l}\text { RockefFolk } \\
(F)\end{array}$ & 36 & $\begin{array}{l}01 / 2000- \\
12 / 2002\end{array}$ & 4680 & $0,57 \%$ & $0,14 \%$ & - \\
\hline $\begin{array}{l}\text { Rock } \\
\text { Hard (F) }\end{array}$ & 42 & $\begin{array}{l}06 / 2001- \\
04 / 2005\end{array}$ & 4872 & $4,16 \%$ & $0,70 \%$ & - \\
\hline Live! (Qc) & 137 & $\begin{array}{l}05 / 1980 \\
03 / 1989\end{array}$ & 3699 & - & - & $1,54 \%$ \\
\hline $\begin{array}{l}\text { Sang frais } \\
\text { (Qc) }\end{array}$ & 17 & $\begin{array}{l}06 / 1998- \\
06 / 2005\end{array}$ & 850 & - & - & $10,70 \%$ \\
\hline \hline Total & 232 & - & 14101 & $4.73 \%$ & $0,84 \%$ & $12,24 \%$ \\
\hline
\end{tabular}

À lui seul, Live! traite près de deux fois plus fréquemment d'artistes français que Rock \&Folk et Rock Hard ne traitent d'artistes québécois. Quant à Sang Frais, on y trouve quasiment 13 fois plus de références à des productions françaises que l'on peut en trouver dans les périodiques français à propos des productions québécoises ${ }^{20}$. Près de 15 fois plus lorsqu'on associe Sang Frais à Live! Les chiffres ne permettent aucun doute. À la lumière de l'échantillon, il existe une profonde dissymétrie entre le dispositif critique français à l'égard des productions québécoises et le dispositif critique québécois à l'égard des productions françaises.

Si l'on rapporte les chiffres français à un niveau canadien, la disproportion se réduit à environ un pour 2,5. Cela signifie que le dispositif critique français est manifestement plus enclin à traiter des productions non québécoises que des productions québécoises (mais on peut également faire l'hypothèse que la presse musicale française accorde une place privilégiée au rock anglophone). Pour en rester à un niveau strictement québécois, et pour donner quelques exemples signifiants, lorsque les périodiques québécois publient 61 chroniques de disques français, les périodiques français publient 11 chroniques de disques québécois. Lorsque les périodiques québécois présentent 30 fanzines français, les périodiques français ne présentent aucun fanzine québécois ${ }^{21}$. Lorsque les périodiques québécois traitent de 79 artistes fran-

20 Il est également intéressant de noter qu'au Québec comme en France, la presse spécialisée se montre plus curieuse que la presse généraliste à l'endroit des productions québécoises ou françaises.

21 Les périodiques français de l'échantillon ne consacrent d'ailleurs pas le moindre espace aux 
çais, les périodiques français traitent de 13 artistes québécois. Lorsque les périodiques québécois réalisent huit interviews ou dossiers présentant des artistes français, les périodiques français en réalisent trois à propos d'artistes québécois.

Le traitement accordé aux productions est également intéressant à observer. Rien de commun en effet entre une "news " strictement informative et une " chronique disque " portant un jugement esthétique par exemple. Dans les périodiques québécois comme dans les périodiques français, les chroniques disques l'emportent généralement sur les simples "news", même si ces dernières peuvent être plutôt abondantes (Sang Frais, Rock Hard). Ce qui signifie qu'en dépit d'une relative faiblesse de traitement journalistique, à l'instar de RockઐFolk, les informations fournies n'en sont pas moins qualitatives. Il est cependant frappant de constater la faiblesse des interviews réalisées de part et d'autre, même si les périodiques québécois en comptent plus du double (8) par rapport aux périodiques français (3), ce qui semble dénoter une certaine distance. L'écart se creuse encore davantage lorsqu'il s'agit des chroniques concerts mettant directement en rapport un journaliste et un artiste ${ }^{22}$. Dans ce cas, les journalistes québécois ont assisté à quatre fois plus de concerts de groupes français (8) que leurs homologues français n'ont assisté aux prestations scéniques de groupes québécois (2). À ce sujet, il est amusant et révélateur de signaler que lorsque RockઐFolk évoque la production scénique au Québec, c'est pour signaler les tournées de Bérurier Noir et de Louise Attaque dans la Belle Province. Une fois encore, l'exemple est éloquent.

Pour féconde qu'elle soit, cette analyse comparative serait cependant incomplète si elle occultait la dynamique des échanges culturels entre le Québec et la France. Car cette dynamique est indissociable de l'environnement qui la rend opérante. C'est la raison pour laquelle il est important d'envisager les données obtenues sous l'angle de la réception critique tout comme de la circulation des productions culturelles de part et d'autre des deux aires francophones.

\section{LA RÉCEPTION CRITIQUE}

Poser la question de la réception qu'un dispositif critique local réserve aux productions provenant d'un espace géographique différent du sien revient évidemment à poser la question des échanges culturels entre ces deux espaces. Ce qui nécessite de l'envisager sous deux aspects essentiels. Côté artistes, la question renvoie au travail promotionnel réalisé en direction de la presse étrangère. Point éminemment difficile à évaluer, mais dont on peut supposer qu'il est effectivement réalisé tant il est improbable que des créateurs - et leurs labels discographiques - se contentent des seuls débouchés médiatiques nationaux. Côté périodiques, la question renvoie très clairement à un rapport

fanzines, d'où qu'ils proviennent.

22 Il faut noter que beaucoup d'interviews se réalisent aujourd'hui par l'intermédiaire du courrier électronique. 
combinant, peu ou prou, curiosité journalistique, goût musical, ligne éditoriale, actualité musicale et bien entendu, intérêt commercial. De ce point de vue, il est pour le moins difficile de soutenir qu'un périodique tel que Rock\&Folk manifeste beaucoup d'intérêt pour la scène rock québécoise. Sa ligne éditoriale reste essentiellement centrée sur les États-Unis et la Grande-Bretagne en leur qualité de lieux historiques de la production rock, mais également sur la France, du fait de son implantation locale. La rubrique « News » est exemplaire de cette focalisation, puisqu'elle couvre essentiellement ces trois territoires. Ainsi, alors qu'au début des années 2000 le label montréalais Constellation devient le pôle mondial de la production post rock, Rock\&Folk ne consacre à Godspeed You! Black Emperor, artiste majeur du label, qu'une seule interview en trois ans. L'exemple en dit long. À l'inverse, un périodique tel que Sang Frais manifeste un intérêt évident pour les productions culturelles en provenance de France. Intérêt d'ailleurs souvent supérieur à la majorité des périodiques français. Ce qui constitue un point d'autant plus surprenant que la ligne éditoriale de ce périodique est explicitement centrée sur les productions québécoises ${ }^{23}$. Cet exemple indique bien que les productions circulent d'un espace à un autre. Toutefois, compte tenu du déséquilibre observé, il est fort probable que les productions circulent plus facilement de la France vers le Québec que dans le sens inverse. En d'autres termes, si le Québec semble effectivement constituer un débouché médiatique pour les productions rock françaises, la France, à l'inverse, ne semble pas nécessairement constituer un débouché médiatique pour les productions rock québécoises. Mais il s'agit là d'une hypothèse impossible à vérifier à partir de mon seul échantillon ${ }^{24}$. Quoiqu'il en soit, ces observations soulignent combien la dimension de la réception est indissociable de la circulation des productions culturelles. Il est donc nécessaire d'y prêter une attention toute aussi soutenue.

\section{LA CIRCULATION DES PRODUCTIONS CULTURELLES}

Les problèmes liés à la circulation des productions culturelles renvoient à de prosaïques questions d'import et d'export. Il est primordial de ne jamais perdre de vue qu'un monde du rock est aussi un marché économique. Il est donc capital de se pencher sur les canaux de distribution des productions culturelles de part et d'autre de l'Atlantique. Il s'agit alors de les identifier à partir des productions culturelles elles-mêmes ${ }^{25}$.

23 À titre d'exemple, l'éditorial inaugural de Sang Frais contient les propos suivants : « Rendez-vous compte, la scène gigote de bons groupes. Pourquoi aller voir ailleurs quand de si bons talents nous entourent? Sans eux, sans vous, sans nous, la scène n'est rien. [...] Je me servirai de ces pages pour vous parler de ce qui se passe au Québec et plus particulièrement à Montréal. " (Sang Frais 1, n⿳0 1 , 1998, p. 3).

24 Vérifier cette hypothèse nécessiterait des investigations plus approfondies sous la forme d'entretiens avec des artistes et des dirigeants de labels. Toutefois, elle m'a été confirmée à deux reprises lors de conversations avec des acteurs importants du monde du rock québécois (dirigeants de labels) qui déclarent privilégier le territoire nord-américain ou britannique.

$25 \mathrm{Il}$ est inutile de chercher à invoquer des problèmes quantitatifs en raison d'une différence d'échelle quant à la démographie artistique entre le Québec et la France. Si la France compte beaucoup d'artistes rock, le Québec en compte également un nombre très élevé. Pour s'en convaincre, il suffit 


\section{Les productions françaises au sein des périodiques québécois}

Si l'on s'intéresse aux artistes français bénéficiant d'une interview ou d'une chronique disque dans les périodiques québécois étudiés, on peut relever les tendances suivantes :

- Dans Live!, l'écrasante majorité (4 sur 5) des artistes français présentés appartient à l'une des quatre multinationales de l'industrie du disque, signifiant par là qu'ils bénéficient de réseaux de distribution planétaires ${ }^{26}$.

- Dans Sang Frais, un peu plus de la moitié (27) des artistes français présentés appartient à des labels indépendants (généralement français ${ }^{27}$ ). Ceux-ci disposent fréquemment de leur propre catalogue de vente par correspondance (Holy Records, Adipocère), voire sont attachés à une structure de distribution indépendante à rayonnement international (Brennus Music, dont le catalogue est distribué en France par Musea, lui-même distribué au Canada par la structure montréalaise Hardel Music; ou encore Osmose Productions, bénéficiant du réseau international du distributeur allemand $S P V$, dont le catalogue est distribué au Canada par la structure montréalaise Fusion III). Dans ce cas, les artistes sont distribués hors France sur la base de contrats de licences $^{28}$ que leurs labels (ou leurs distributeurs) établissent le plus souvent avec des structures homologues à l'étranger pour lesquelles ils effectuent quelquefois le même type de travail. L'autre frange d'artistes, minoritaire (25), recourt à l'autodistribution de sa propre initiative, ou par l'intermédiaire d'un petit label. Dans ce cas, il s'agit souvent d'un travail militant par lequel les artistes et leurs labels alimentent le réseau mondial de la scène musicale à laquelle ils appartiennent. Il s'agit d'un procédé employé par la totalité des fanzines que l'on trouve dans les pages de Sang Frais.

\section{Les productions québécoises au sein des périodiques français}

Si l'on s'intéresse aux artistes québécois bénéficiant d'une interview ou d'une chronique disque dans les périodiques français étudiés, on peut relever les tendances suivantes :

d'une simple visite sur le site Québec Punk Scène qui recense pas moins de $\mathbf{4 4 2}$ formations au sein du genre musical punk. Voir : http://www.quebecpunkscene.net/ (consulté le 27 juillet 2005).

26 Mario d'Angelo rappelle que « la fonction de distribution [...] est la condition sine qua non pour être une major. Elle confere l'accès au marché mondial en mettant le distributeur dans la position de maîtriser l'incertitude liée aux différences des marchés nationaux et territoriaux (structuration des canaux de diffusion, "goûts" spécifiques des consommateurs, rôles des faiseurs d'opinion, etc.) " (D'Angelo 1998, 24).

27 Il est intéressant de noter que le groupe parisien Fate est sous contrat avec le label montréalais Galy Records. Ả l'inverse, le groupe montréalais Frozen Shadows est sous contrat avec le label français Holy Records.

28 Le système de licence permet à un disque produit à l'étranger d'acquérir un statut domestique dans le pays qui le distribue; ce qui a une incidence directe sur le prix du disque, à l'inverse du disque importé dont le prix est évidemment bien plus élevé sur le marché local. 
- Dans RockઐFolk, la présence de Leonard Cohen se justifie par son appartenance à l'une des cinq multinationales de l'industrie du disque (Sony/Columbia), signifiant par là que son œuvre bénéficie d'un réseau de distribution planétaire, tandis que celle de A Silver Mt Zion se justifie par sa présence dans le catalogue du distributeur français Chronowax.

- Dans Rock Hard, la majorité (6) des artistes québécois présentés appartient à d'importantes maisons de disques spécialisées dans le metal (Roadrunner, Century Media, Nuclear Blast) ou de labels moins importants (Divine Recordings, label de Sharon Osbourne ${ }^{29}$, ou Chophouse Records, label de Jason Newsted ${ }^{30}$ ) bénéficiant de réseaux de distribution indépendants ou non, mais à tout le moins planétaires. L'autre frange (4) fonctionne d'une part avec des distributeurs français (Musea, Holy Records) et d'autre part sur la base de l'autodistribution de leur propre initiative ou par l'intermédiaire d'un petit label. Dans ce cas, il s'agit souvent d'un travail militant par lequel les artistes et leurs labels alimentent le réseau mondial de la scène musicale à laquelle ils appartiennent.

Au final, il apparaît tout d'abord que, en France comme au Québec, les disques des artistes cités bénéficient majoritairement de services de distribution locaux en capacité d'orchestrer des campagnes de promotion ciblées en direction de la presse spécialisée. Dans un second temps, il faut également observer qu'une frange non négligeable d'artistes et de producteurs de fanzines réalise ce travail de leur propre initiative. Leurs connaissances personnelles conduisent ces derniers à constituer leur propre réseau au sein duquel il leur est possible de faire circuler leurs productions et d'y trouver un moyen des les exposer par l'intermédiaire de la presse spécialisée. En résumé, la circulation des productions culturelles au sein de l'espace franco-québécois est donc tributaire à la fois de réseaux de distribution commerciaux tout autant que de réseaux communautaires et affinitaires ("scènes musicales"). Si ce circuit produit des débouchés d'une efficacité très variable, il faut également considérer le fait que les producteurs culturels ciblent la destination de leurs productions en fonction de l'intérêt qu'elle revêt à leurs yeux. Et qu'à ce titre, la France comme le Québec peuvent tout simplement représenter un territoire plus ou moins pertinent qu'un autre.

\section{Conclusion}

La réception des productions québécoises au sein des périodiques français comparativement à la réception des productions françaises au sein des périodiques québécois apparaît nettement déséquilibrée. Il est difficile d'imputer cette dissymétrie à un problème de distribution quelconque. La circulation des productions culturelles reste parfaitement fluide, dans un sens comme

29 Epouse d'Ozzy Osbourne, chanteur de Black Sabbath dont la famille est désormais mondialement connue grâce à ses frasques relayées par un reality-show à succès diffusé sur MTV, The Osbournes.

30 Ancien bassiste, multimillionnaire, du groupe Metallica. 
dans l'autre. Ce qui donne à penser que les périodiques québécois se montrent a priori plus réceptifs aux productions françaises que l'inverse. Toutefois, si l'axe France-Québec semble offrir un rapport favorable aux productions culturelles françaises comparativement aux productions culturelles québécoises, rien n'indique véritablement que ces dernières souffrent de ce déficit. Car si le dispositif critique français étudié dans le cadre de cet article se caractérise de manière incontestable par une certaine forme d'ethnocentrisme ${ }^{31}$, il est également envisageable de considérer que les productions rock québécoises ne visent pas nécessairement la France en tant que territoire de réception ${ }^{32}$. Une hypothèse qui, si on l'accepte, conduit alors à se demander si du point de vue des producteurs culturels québécois, c'est la perception de cet ethnocentrisme qui est à l'origine de ce déséquilibre ou alors si la France n'apparaît tout simplement pas à leurs yeux comme un territoire à privilégier ${ }^{33}$. Voire les deux à la fois. La question reste posée et mérite des développements ultérieurs. Cette étude est donc avant tout à considérer comme la première étape d'un travail exploratoire.

\section{RÉFÉRENCES}

André, Daniel. 1993. « Rôle et statut social des artistes de la chanson populaire à travers le discours journalistiques des années 80 ». Dans En avant la chanson!, sous la dir. de Robert Giroux, 149-75. Montréal : Tryptique.

Angers, Stéphanie et Gérard Fabre. 2004. Echanges intellectuels entre la France et le Québec (1930-2000) : Les réseaux de la revue Esprit avec La relève, Cité libre, Parti pris et Possibles. Québec : Presses de l'Université Laval; Paris : L'Harmattan.

Bennett, Andy et Richard A. Peterson, dir. 2004. Music Scenes : Local, Translocal, and Virtual. Nashville, TN : Vanderbilt University Press.

Bonnetti, Erwan. 2005. "Musik ». Québec Scope Magazine 8, nº 7 (août) : 22. Callon, Michel. 1986. "Eléments pour une sociologie de la traduction : La domestication des coquilles Saint-Jacques et des marins-pêcheurs dans la baie de Saint-Brieuc ". L'Année Sociologique 36 : 170-207.

Casanova, Pascale. 1999. La République mondiale des Lettres. Paris : Seuil.

D'Angelo, Mario. 1998. "L'impitoyable industrie du disque : Qui contrôle la musique? ". Le monde diplomatique, $\mathrm{n}^{\circ} 531$ (juin) : 24-25.

De Surmont, Jean-Nicolas. 1996. "Regards croisés entre la France et le Québec : Pour une approche diachronique des transferts relatifs à la

31 Ce qui va dans le sens de l'analyse de la vedette québécoise Stefie Shock, qui déplore à propos de la France que " [ [']import-export se fait pratiquement dans un sens. Nous, on reçoit bien plusieurs de leurs artistes, mais le retour du balancier ne se fait pas vraiment " (propos rapportés dans Bonnetti 2005, 22).

32 Se pose ici une question linguistique évidente. Les groupes d'expression anglophone sont certainement moins focalisés sur la France que ne le sont les groupes d'expression francophone.

33 Dans ce cas, la situation serait inverse à celle qu'observe Josée Vincent (1997) à propos de la circulation du livre québécois, pour lequel la diffusion en France équivaut à une forme de légitimation au sein du monde francophone. Ce qui accréditerait l'idée selon laquelle les artistes rock québécois recherchent une légitimation ailleurs qu'en France. 
chanson ». Dans La chanson: Carrières et société, sous la dir. de Robert Giroux, 193-220. Montréal : Tryptique.

Durkheim, Emile. 1894. Les règles de la méthode sociologique. Coll. : "Champs ». Paris : Flammarion, 1988.

Étienne, Samuel. 2003. " "First \& Last \& Always" : les valeurs de l'éphémère dans la presse musicale alternative ". Copyright Volume! 2, $\mathrm{n}^{\circ} 1: 5-34$.

Fabre, Gérard. 2004. "Un arc transatlantique et sa tangente ou comment se dessine un réseau intellectuel franco-québécois? ". Globe : Revue internationale d'études québécoises $7, \mathrm{n}^{\circ} 1: 43-78$.

Hein, Fabien. 2003a. Hard Rock, Heavy Metal, Metal : Histoire, cultures et pratiquants. Coll. "Musique et société ». Clermont-Ferrand/Paris : Mélanie Séteun/Irma.

Hein, Fabien. 2003b. "Les fanzines rock et leurs rédacteurs en Lorraine ». Ethnographiques.org, $\mathrm{n}^{\mathrm{o}} 3$ (avril). http://www.ethnographiques.org/ 2003/Hein.html (consulté le 27 janvier 2006).

Hein, Fabien. 2006. Le monde du rock: Ethnographie du réel. Coll. « Musique et société ». Clermont-Ferrand/Paris : Mélanie Séteun/Irma.

Hennion, Antoine. 1993. La passion musicale: Une sociologie de la médiation. Paris : Métailié.

Langlois, Simon. 2002. "Présentation: Au Québec et ailleurs : Comparaisons de sociétés ". Recherches Sociographiques 43, $\mathrm{n}^{\circ} 1$ (janvier-avril) : 9-18.

Latour, Bruno. 1989. La science en action. Paris : La découverte.

Manœuvre, Philippe. 1985. L'enfant du rock. Paris : J.-C. Lattès.

Paoli, Létitia. 1993. "Etudes des motivations des troupes de théâtre amateur françaises et québécoises à augmenter leur implication internationale ". Mémoire de maîtrise, Université Laval, Québec.

Rudent, Catherine. 2000. "Le discours sur la musique dans la presse française : L'exemple des périodiques spécialisés en 1993 ». Thèse de doctorat, Université de Paris-Sorbonne.

Sartori, Giovanni. 1994. "Bien comparer, mal comparer ". Revue Internationale de Politique Comparée $1, \mathrm{n}^{\circ} 1: 19-36$.

Straw, Will. 1991. "Systems of Articulation, Logics of Change : Communities and Scenes in Popular Music ». Cultural Studies 5, $\mathrm{n}^{\circ} 3$ (octobre) : 368-88.

Teillet, Philippe. 2002. "Les cultes musicaux : La contribution de l'appareil de commentaires à la construction des cultes, l'exemple de la presse rock ». Dans Les cultes médiatiques: Culture fan et ouvres cultes, sous la dir. de Philippe Le Guern, 309-41. Coll. : "Le lien social ». Rennes : Presses Universitaires de Rennes.

Vigour, Cécile. 2005. La comparaison dans les sciences sociales: Pratiques et méthodes. Coll. : « Repères ». Paris : La découverte.

Vincent, Josée. 1997. Les tribulations du livre québécois en France (1959-1985). Québec : Nuit Blanche Éditeur.

Willey, Mireille. 1994. "Le rock à travers la presse spécialisée ». Dans Le rock. Aspects esthétiques, culturels et sociaux, sous la dir. d'Anne-Marie Gourdon, 181-212. Coll. : " Arts du spectacle ». Paris : CNRS. 
Sites Web Des Périodiques Cités

Bazooka: <http://www.piratesrecords.com/magazines.htm>

Emoragei : <http://www.emorageimagazine.com>

Longueur d'Ondes : <http://www.longueurdondes.com >

Rien à Déclarer : <http://radzine.com $>$

Rock Hard : <http://www.rockhard.fr >

Rock GFolk : <http://www.rocknfolk.com>

Sang Frais : <http://www.sangfrais.com>

\title{
RESUME
}

À partir d'une étude comparative chiffrée, cet article vise à porter un regard croisé sur la réception accordée par la presse musicale française aux productions rock québécoises/canadiennes, et inversement, à la réception accordée par la presse musicale québécoise aux productions rock françaises. À condition de ne pas séparer les données obtenues des réalités du travail de la réception critique tout comme de la circulation des productions culturelles, cette démarche permet d'évaluer la dynamique des échanges culturels entre les deux aires francophones.

\begin{abstract}
This paper is a cross-cultural study comparing statistical data about the reception to Québec/Canadian rock productions by the French musical press and, conversely, the reception to French rock productions by the Québec musical press. Maintaining a link between the data and the reality of dealing with critical reception as well as the circulation of cultural productions is the key to this approach that makes an evaluation of the dynamic of the cultural exchange between the two francophones zones possible.
\end{abstract}

